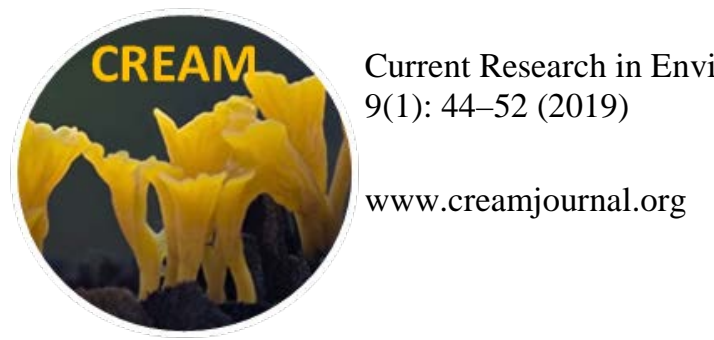

\title{
Hierarchically weighted principal component analysis evaluation of antifungal azoles inhibitory potency on lanosterol-14a-demethylase in Candida albicans
}

\author{
Sumalapao DEP ${ }^{1,2, *}$ and Gloriani NG $^{1}$
}

${ }^{1}$ Department of Medical Microbiology, College of Public Health, University of the Philippines Manila, Manila, Philippines

${ }^{2}$ Biology Department, College of Science, De La Salle University, Manila, Philippines

Sumalapao DEP, Gloriani NG 2019 - Hierarchically weighted principal component analysis evaluation of antifungal azoles inhibitory potency on lanosterol-14 $\alpha$-demethylase in Candida albicans. Current Research in Environmental \& Applied Mycology (Journal of Fungal Biology) 9(1), 44-52, Doi 10.5943/cream/9/1/5

\begin{abstract}
Hierarchically weighted principal component analysis method was employed to assess the inhibitory potency of different antifungal azoles to Candida albicans lanosterol-14 $\alpha$-demethylase. Information regarding the molecular properties and biological activity of the antifungal azoles was utilized for evaluation. The molecular weight, complexity, heavy atom count, and rotatable count were identified as the most significant variables describing the inhibitory potency of the antifungal drugs. Newer generation drugs including itraconazole and fluconazole were identified to have better inhibitory potency than bifonazole and clotrimazole. The present study illustrated a method providing an effective approach in classifying antifungal azoles based on molecular properties and biological activity for possible synthesis of newer generation antifungal drugs for a better and more effective treatment of fungal infections.
\end{abstract}

Key words - Antifungal azoles - Candida albicans - Cytochrome P450 enzyme system - Inhibitory potency - Itraconazole - Molecular complexity - Molecular weight - Rotatable count

\section{Introduction}

Nearly every part of the gastrointestinal tract, from the oral cavity up to the perianal tissues, epidermis, and the vulvovaginal region, is colonized by Candida albicans (Pfaller \& Diekema 2007). However, C. albicans is notorious for causing candidiasis, with the potential of becoming a systemic infection, subsequently provoking a much more serious medical condition (Rekha \& Visyasagar 2013). There is an increase in the frequency of infections globally due to pathogenic microorganisms resulting to increased cases of morbidity and mortality. Candida albicans is the most common human fungal pathogen, and mortality from C. albicans infection is still unacceptably high (Pfaller \& Diekema 2007). These mycoses are commonly found in medical practice in increasing frequency, mainly because of broad-spectrum antibiotic therapy (Ghannoum \& Rice 1999). In the therapeutic management of systemic fungal infections and local mycoses, azoles remain the currently used antifungal agents (Zonios \& Bennett 2008) and have been an important pharmacophore and privileged structure in medicinal chemistry. Azoles inhibit fungal lanosterol-14 $\alpha$-demethylase, an important enzyme in the synthesis of ergosterol, a major fungal plasma membrane component 
(Zampieri et al. 2007). Lanosterol-14 $\alpha$-demethylase or CYP51 (cytochrome P450 isoenzyme 51), a family of phylogenetic highly conserved monooxygenases, was identified in humans, plants, animals, fungi, and mycobacteria (Lepesheva \& Waterman 2004). It is a rate limiting step in the production of follicular fluid-meiosis activating steroid, a precursor in mammalian and yeast sterol biosynthesis (Rozman \& Waterman 1998), which subsequently leads in the synthesis of ergosterol, in the case of fungi. This explains the mechanism of azoles as fungicides inhibiting CYP51 which results in the absence of ergosterol, an essential fungal cell membrane component. However, C. albicans infections detect several problems including relapse of the fungal infections, resistance to commonly used antifungals, and availability of potent and inexpensive effective antifungal agents. Hence, the search for new and effective synthetic inhibitors of $C$. albicans is an actual and important task for basic science and clinical medicine.

In this study, the inhibitory potency of different antifungal azoles to lanosterol-14 $\alpha$ demethylase of $C$. albicans was assessed using hierarchically weighted principal component analysis method. Molecular descriptors, geometrical properties, and electronic parameters such as molecular weight, rotatable count, complexity, hydrogen bond donor and acceptor counts, topological polar surface area, lipophilicity, heavy atom count, and undefined atom stereocenter were considered as indices in the evaluation of the biological activity $\left(\mathrm{IC}_{50}\right)$ of currently available antifungal agents. The experimental measurement of bioactivity of compounds is difficult, time-consuming, and expensive. Hence, attempts such as statistical modeling were done to predict these biological activities (Sumalapao et al. 2018). The present study illustrated the utility of hierarchically weighted principal component analysis method as an effective approach in classifying antifungal azoles, based on molecular properties and biological activity for possible synthesis of newer generation antifungal drugs for a better and more effective treatment of fungal infections.

\section{Materials \& Methods}

\section{Biological Activity of Antifungal Azoles}

Biological information on the inhibitory potencies $\left(\mathrm{IC}_{50}\right)$ of the nineteen (19) different antifungal agents, thirteen (13) of which were used in agriculture and six (6) in human medicine, on C. albicans CYP51 was obtained from literature (Trosken et al. 2006). The thirteen fungicides used agriculturally were bitertanol, cyproconazole, epoxiconazole, flusilazole, hexaconazole, imazalil, myclobutanil, penconazole, prochloraz, propiconazole, tebuconazole, triadimefon, and triadimenol. Bifonazole, clotrimazole, fluconazole, itraconazole, ketoconazole, and miconazole were the six human medicine antimycotics.

\section{Structural and Molecular Properties of Antifungal Azoles}

Molecular descriptors, geometrical properties, and electronic parameters of the azoles evaluated in this study include molecular weight, rotatable count, complexity, hydrogen bond donor and acceptor counts, topological polar surface area, lipophilicity, heavy atom count, and undefined atom stereocenter. Information regarding these descriptors of the nineteen antifungal azoles (Table 1) was obtained from the National Center for Biotechnology Information (NCBI) databases.

\section{Principal component analysis}

In this study, principal component analysis was employed primarily to describe the similarity among antifungal agents and the correlations among the molecular descriptors. This multivariate statistical approach summarizes and visualizes information present in the data set including a small number of new variables (principal components) which are uncorrelated linear combinations with maximal variance (Lebart et al. 1984, Jolliffe 2002, Hastie et al. 2009). In this method, a given set of correlation variables is transformed into another set of uncorrelated variables by linear transformation, and subsequently these new variables are arranged in order of decreasing variance. The total variance of the variables is kept constant, such that the first principal component assumes the largest variance, with subsequent components in decreasing variance not related to the previous 
principal components. In this study, all statistical analyses were carried out using STATA ${ }^{\circledR}$ at $5 \%$ level of significance.

Table 1 Molecular properties of the different antifungal azoles (National Center for Biotechnology Information 2018a, b, c, d, e, f, g, h, i, j, k, l, m, n, o, p, q, r, s).

\begin{tabular}{|c|c|c|c|c|c|c|c|c|c|}
\hline \multirow{2}{*}{ Antifungal Azole } & \multicolumn{9}{|c|}{ Molecular Properties } \\
\hline & $\operatorname{logmw}$ & hbdc & hbac & rc & logcomp & logtpsa & $\mathrm{x} \log \mathrm{P}$ & loghac & uasc \\
\hline Bifonazole & 2.492 & 0 & 1 & 4 & 2.559 & 1.250 & 4.8 & 1.380 & 1 \\
\hline Bitertanol & 2.528 & 1 & 4 & 6 & 2.600 & 1.780 & 4.2 & 1.398 & 2 \\
\hline Clotrimazole & 2.538 & 0 & 1 & 4 & 2.598 & 1.250 & 5.0 & 1.398 & 0 \\
\hline Cyproconazole & 2.465 & 1 & 3 & 5 & 2.520 & 1.707 & 2.9 & 1.301 & 2 \\
\hline Epoxiconazole & 2.518 & 0 & 4 & 4 & 2.624 & 1.635 & 3.2 & 1.362 & 2 \\
\hline Fluconazole & 2.486 & 1 & 7 & 5 & 2.554 & 1.912 & 0.4 & 1.342 & 0 \\
\hline Flusilazole & 2.499 & 0 & 4 & 4 & 2.522 & 1.487 & 3.7 & 1.342 & 0 \\
\hline Hexaconazole & 2.497 & 1 & 3 & 6 & 2.489 & 1.707 & 3.7 & 1.301 & 1 \\
\hline Imazalil & 2.473 & 0 & 2 & 6 & 2.464 & 1.431 & 3.8 & 1.279 & 1 \\
\hline Itraconazole & 2.849 & 0 & 9 & 11 & 3.049 & 2.004 & 5.7 & 1.690 & 1 \\
\hline Ketoconazole & 2.725 & 0 & 6 & 7 & 2.866 & 1.839 & 4.3 & 1.556 & 0 \\
\hline Miconazole & 2.619 & 0 & 2 & 6 & 2.620 & 1.431 & 5.3 & 1.398 & 1 \\
\hline Myclobutanil & 2.461 & 0 & 3 & 6 & 2.538 & 1.736 & 2.9 & 1.301 & 1 \\
\hline Penconazole & 2.454 & 0 & 2 & 5 & 2.401 & 1.487 & 4.4 & 1.255 & 1 \\
\hline Prochloraz & 2.576 & 0 & 3 & 6 & 2.576 & 1.676 & 4.6 & 1.362 & 0 \\
\hline Propiconazole & 2.534 & 0 & 4 & 5 & 2.576 & 1.692 & 3.5 & 1.342 & 2 \\
\hline Tebuconazole & 2.488 & 1 & 3 & 6 & 2.513 & 1.707 & 3.7 & 1.322 & 1 \\
\hline Triadimefon & 2.468 & 0 & 4 & 5 & 2.529 & 1.756 & 2.8 & 1.301 & 1 \\
\hline Triadimenol & 2.471 & 1 & 4 & 5 & 2.481 & 1.780 & 3.1 & 1.301 & 2 \\
\hline
\end{tabular}

logmw (g/mol): logarithm of molecular weight; hbdc: hydrogen bond donor count; hbac: hydrogen bond acceptor count; rc: rotatable count; logcomp: logarithm of complexity; logtpsa: logarithm of topological polar surface area; xlogP: lipophilicity; loghac: logarithm of heavy atom count; uasc: undefined atom stereocenter

\section{Results}

Descriptive measures of the nineteen different antifungal agents tested for their inhibitory potencies on C. albicans CYP51 and their respective molecular properties (molecular weight, rotatable count, complexity, hydrogen bond donor and acceptor counts, topological polar surface area, lipophilicity, heavy atom count, and undefined atom stereocenter) as indices of biological activity evaluation were calculated (Table 2). The range of inhibitory potency is from 0.039 to 0.350 $\mu \mathrm{M}$ among the identified antifungal drugs on CYP51 of C. albicans.

Table 2 Biological activity and molecular indices of the antifungal azoles.

\begin{tabular}{llll}
\hline Index & Range & Mean & Standard deviation \\
\hline $\mathrm{IC}_{50}$ & $0.039-0.350$ & 0.132 & 0.094 \\
logmw & $2.454-2.849$ & 2.534 & 0.101 \\
hbdc & $0.000-1.000$ & 0.316 & 0.478 \\
hbac & $1.000-9.000$ & 3.632 & 1.978 \\
rc & $4.000-11.000$ & 5.578 & 1.575 \\
logcomp & $2.401-3.049$ & 2.583 & 0.147 \\
logtpsa & $1.250-2.004$ & 1.646 & 0.206 \\
xlogP & $0.400-5.700$ & 3.789 & 1.175 \\
loghac & $1.255-1.690$ & 1.365 & 0.103 \\
uasc & $0.000-2.000$ & 1.000 & 0.745 \\
\hline
\end{tabular}

$\mathrm{IC}_{50}(\mathrm{uM})$ : inhibitory concentration (Trosken et al. 2006)

Loading values of each evaluation index in the principal components were calculated using STATA $^{\odot}$ at $5 \%$ level of significance. Based on principal component contribution rate, the first, second, and third principal components accounted for $51.79 \%$, 25.50\%, and $11.38 \%$, respectively (Table 3). The indices identified in the first principal component are the molecular weight, rotatable 
count, complexity, and heavy atom count. These indices in the first principal component describe the molecular complexity of the antifungal agents. Moreover, the indices included in the second principal component are the hydrogen bond donor count, topological polar surface area, and lipophilicity. The third principal component included lipophilicity and undefined atom stereocenter. Therefore, the biological activity of the antifungal agents was mainly influenced by the first and second principal components consisting of molecular weight, rotatable count, complexity, and heavy atom count, followed by hydrogen bond donor count, topological polar surface area, and lipophilicity.

Table 3 Eigenvectors and loading values of the principal components.

\begin{tabular}{lccc}
\hline & \multirow{2}{*}{ Index } & \multicolumn{2}{c}{ Principal components } \\
\cline { 2 - 3 } & $\mathbf{1}$ & $\mathbf{2}$ & $\mathbf{3}$ \\
\hline logmw & 0.4475 & -0.1305 & 0.0358 \\
hbdc & -0.0962 & 0.5000 & 0.1945 \\
hbac & 0.3556 & 0.3610 & -0.2115 \\
rc & 0.4016 & 0.0777 & 0.1732 \\
logcomp & 0.4481 & -0.0550 & 0.0144 \\
logtpsa & 0.2531 & 0.5235 & -0.0338 \\
XlogP & 0.1868 & -0.5038 & 0.4114 \\
loghac & 0.4421 & -0.1051 & 0.0163 \\
uasc & -0.0871 & 0.2339 & 0.8457 \\
\hline Eigenvalue & 4.6608 & 2.2952 & 1.0242 \\
Difference & 2.3657 & 1.2709 & 0.4919 \\
Proportion & 0.5179 & 0.2550 & 0.1138 \\
Cumulative & 0.5179 & 0.7729 & 0.8867 \\
\hline
\end{tabular}

When hierarchically weighted principal component analysis method was employed in evaluating the inhibitory potency of the different antifungal azoles, resulting calculated comprehensive scores ranged from 2.38 to 6.43 (Table 4), with higher comprehensive scores identified in the newer generations of antifungal agents compared with the older generations. Moreover, comprehensive scores for antifungal agents intended as human medicines for mycoses are at least 4.00 for newer generation agents and at most 3.00 for the older antifungal drugs, while azoles for agricultural purposes have comprehensive scores ranging between 3.00 to 4.00 (Table 4).

Table 4 Comprehensive evaluation of the antifungal azoles.

\begin{tabular}{|c|c|c|c|c|c|c|}
\hline \multirow{2}{*}{ Antifungal Drug } & \multicolumn{4}{|c|}{ Principal Component Score } & \multirow{2}{*}{ Rank } & \multirow{2}{*}{$\mathbf{I C}_{50}$} \\
\hline & Component 1 & Component 2 & Component 3 & Comprehensive & & \\
\hline Itraconazole & 11.0063 & 1.4266 & 3.1907 & 6.4271 & 1 & 0.039 \\
\hline Fluconazole & 7.3476 & 3.8262 & 0.1646 & 4.7998 & 2 & 0.051 \\
\hline Ketoconazole & 8.1369 & 0.9626 & 1.7690 & 4.6609 & 3 & 0.064 \\
\hline Bitertanol & 6.7464 & 0.7597 & 3.4193 & 4.0768 & 4 & 0.059 \\
\hline Triadimenol & 6.2233 & 1.3138 & 2.9667 & 3.8957 & 5 & 0.330 \\
\hline Propiconazole & 6.3124 & 0.5664 & 3.1313 & 3.7700 & 6 & 0.150 \\
\hline Triadimefon & 6.2432 & 0.9526 & 1.9976 & 3.7036 & 7 & 0.130 \\
\hline Tebuconazole & 6.3006 & 0.6124 & 2.3679 & 3.6887 & 8 & 0.350 \\
\hline Hexaconazole & 6.2842 & 0.6124 & 2.3679 & 3.6802 & 9 & 0.066 \\
\hline Cyproconazole & 5.8822 & 1.0154 & 2.8845 & 3.6336 & 10 & 0.100 \\
\hline Myclobutanil & 6.2899 & 0.5310 & 2.0388 & 3.6249 & 11 & 0.140 \\
\hline Epoxiconazole & 5.9337 & 0.6880 & 3.0079 & 3.5908 & 12 & 0.220 \\
\hline Prochloraz & 6.3856 & -0.3572 & 1.8924 & 3.4314 & 13 & 0.098 \\
\hline Flusilazole & 5.8708 & 0.3585 & 1.5222 & 3.3051 & 14 & 0.085 \\
\hline Imazalil & 5.8969 & -0.4431 & 2.4090 & 3.2151 & 15 & 0.082 \\
\hline Miconazole & 6.0850 & -1.1988 & 3.0261 & 3.1901 & 16 & 0.072 \\
\hline Penconazole & 5.4482 & -0.7162 & 2.6559 & 2.9412 & 17 & 0.076 \\
\hline Bifonazole & 4.8339 & -1.4026 & 2.8204 & 2.4668 & 18 & 0.300 \\
\hline Clotrimazole & 4.8796 & -1.5034 & 2.0570 & 2.3779 & 19 & 0.091 \\
\hline
\end{tabular}

$\mathrm{IC}_{50}(\mathrm{uM})$ : inhibitory concentration (Trosken et al. 2006) 
Among the molecular properties of antifungal azoles evaluated in this study, molecular weight has been identified to have a positive correlation with the complexity, rotatable count, heavy atom and hydrogen bond acceptor counts (Table 5). In the correlation analysis, topological polar surface area is directly correlated with hydrogen bond acceptor and rotatable counts (Table 5). Lipophilicity of the antifungal azoles was positively correlated with molecular weight and heavy atom count but negatively correlated with hydrogen bond donor count (Table 5). On the other hand, molecular complexity is directly correlated with hydrogen bond acceptor count and rotatable count (Table 5).

Table 5 Correlation coefficients among the different molecular indices of the antifungal azoles.

\begin{tabular}{llllllllll}
\hline & $\operatorname{logmw}$ & hbdc & hbac & rc & logcomp & logtpsa & xlogP & loghac & uasc \\
\hline logmw & 1.0000 & -0.3091 & $0.6196^{* *}$ & $0.7950^{* *}$ & $0.9560^{* *}$ & 0.3604 & $0.5590^{*}$ & $0.9616^{* *}$ & -0.2281 \\
hbdc & -0.3091 & 1.0000 & 0.1300 & -0.0350 & -0.2717 & 0.4053 & $-0.4689^{*}$ & -0.2537 & 0.3121 \\
hbac & $0.6196^{* *}$ & 0.1300 & 1.0000 & $0.6431^{* *}$ & $0.6989^{* *}$ & $0.8492^{* *}$ & -0.2240 & $0.6465^{* *}$ & -0.0754 \\
rc & $0.7950^{* *}$ & -0.0350 & $0.6431^{* *}$ & 1.0000 & $0.7486^{* *}$ & $0.5910^{* *}$ & 0.3788 & $0.7291^{* *}$ & -0.0473 \\
logcomp & $0.9560^{* *}$ & -0.2717 & $0.6989^{* *}$ & $0.7486^{* *}$ & 1.0000 & 0.4383 & 0.4162 & $0.9832^{* *}$ & -0.1601 \\
$\operatorname{logtpsa}$ & 0.3604 & 0.4053 & $0.8492^{* *}$ & $0.5910^{* *}$ & 0.4383 & 1.0000 & -0.3858 & 0.3490 & 0.1553 \\
xlogP & $0.5590^{*}$ & $-0.4689^{*}$ & -0.2240 & 0.3788 & 0.4162 & -0.3858 & 1.0000 & $0.5002^{*}$ & -0.0698 \\
loghac & $0.9616^{* *}$ & -0.2537 & $0.6465^{* *}$ & $0.7291^{* *}$ & $0.9832^{* *}$ & 0.3490 & $0.5002^{*}$ & 1.0000 & -0.2157 \\
uasc & -0.2281 & 0.3121 & -0.0754 & -0.0473 & -0.1601 & 0.1553 & -0.0698 & -0.2157 & 1.0000 \\
\hline
\end{tabular}

$0.05\left(^{*}\right)$ and $0.01(* *)$ level of significance

\section{Discussion}

When principal component analysis method was employed, the present study identified itraconazole as the most potent among the antifungal azoles. This is in complete agreement with the findings of Trosken et al. (2006). Among the molecular properties of antifungal azoles evaluated in this study, molecular weight has been identified to have a positive correlation with the complexity, rotatable count, heavy atom and hydrogen bond acceptor counts. In small molecule drug discovery, molecular weight is an important property (Meanwell 2011, Leeson 2016) commonly monitored during the compound optimization steps, as this can influence several molecular events including absorption, interactions with on- and off-targets, blood brain barrier penetration, and bile elimination rate (Gleeson 2008). On the other hand, molecular complexity is directly correlated with hydrogen bond acceptor count and rotatable count. Molecular complexity accounts the fraction of $s p^{3}$ hybridized carbons, the number of stereocenters, and the number of rings and aromatic rings. Aromaticity increases $\log \mathrm{p}$ and affinity for albumin, but it decreases the free unbound form species of the molecule and its aqueous solubility (Ritchie \& Macdonald 2009). Moreover, a molecule with more than three aromatic rings was associated with increased risk of toxicity (herg and cyp inhibition) and had poorer compound developability (Lovering et al. 2009). Complexity indices based on the first-order topological neighborhood of atoms (Roy et al. 1984) considered the number of hydrogen atoms and the presence of double and triple bonds (Hendrickson et al. 1987). Because of specific binding events in the complete ligand molecule, moderately complex structures were preferred lead compounds (Hann et al. 2001). Molecular complexity is related with biological activity (Hann \& Oprea 2004) and molecular pharmacologic properties including solubility, oral bioavailability, permeability, promiscuity, and clinical progression (Lovering 2013).

In the present study, hydrogen bond donor count was one of the primary indices included in the second principal component. Hydrogen bond donors and acceptors were identified to be related with the polarity and permeability of compounds (Alex et al. 2011). Hydrogen bond donor count corresponds to the total number of hydrogen atoms bonded to any oxygen and nitrogen atoms without negative charge in the molecule, while hydrogen bond acceptor count sums up any oxygen, fluorine, and nitrogen atoms excluding pyrrolyl nitrogen form, higher oxidation states, and positive formal charge. Among the marketed oral drugs, molecular weight and hydrogen bond acceptor have significantly increased, whereas lipophilicity and hydrogen bond donor have shown relatively limited changes over time (Wenlock et al. 2003, Leeson \& Davis 2004), suggesting that hydrogen bond donor count may be more important than hydrogen bond acceptor count (Baell et al. 2013, Leeson 2016) as 
hydrogen bond donor count enhanced bioavailability and membrane permeability (Leeson \& Davis 2004).

Moreover, lipophilicity of the antifungal azoles was positively correlated with molecular weight and heavy atom count but negatively correlated with hydrogen bond donor count. This index, an important determinant in optimizing the pharmacodynamic and pharmacokinetic properties of molecules in medicinal chemistry and in computer-aided drug design (Kujawski et al. 2012), influences molecular permeability, absorption, bioavailability, metabolism, toxicity, distribution, potency, and excretion parameters. Metabolism and solubility are more likely compromised by high lipophilic compounds, whereas low lipophilicity decreases permeability (Waring 2010). Highly lipophilic compounds resulted in promiscuity and toxicity problems such as cytochrome p450 and herg inhibitions (Gleeson 2008, Waring 2010). Lipophilicity governs the kinetics and dynamics of drug action since optimal lipophilicity range, along with low polar surface area and molecular weight, results in better intestinal absorption of the drug via passive diffusion (Kubinyi 1979, Dearden 1985).

In the correlation analysis, topological polar surface area is directly correlated with hydrogen bond acceptor and rotatable counts. Topological polar surface area, an important parameter in predicting drug transport properties, correlates well with Caco-2 monolayers permeability, human intestinal absorption, and blood-brain barrier penetration (Ertl et al. 2000), oral bioavailability and permeability estimation (Leeson 2016). When combined with rotatable bond count, topological polar surface area reflects molecular flexibility. Increase in the number of rotatable bonds and topological polar surface area decreases the bioavailability of a compound (Veber et al. 2002).

In conclusion, inhibitory potency of different antifungal azoles to lanosterol-14 $\alpha$-demethylase of Candida albicans was assessed using hierarchically weighted principal component analysis method. Molecular weight, complexity, heavy atom count, and rotatable count were identified as the most significant molecular properties describing the inhibitory potency of these antifungal drugs. The present study illustrated a method providing an effective approach in classifying antifungal drugs based on molecular properties and biological activity for possible synthesis of newer generation antifungal drugs for a better and more effective treatment of fungal infections.

\section{References}

Alex A, Millan D, Perez MA, Whitlock GA. 2011 - Intramolecular hydrogen bonding to improve membrane permeability and absorption in beyond rule of five chemical space. Medicinal Chemistry Communication 2, 669-674.

Baell J, Congreve M, Leeson P, Abad-Zapatero C. 2013 - Ask the experts: past, present and future of the rule of five. Future Medicinal Chemistry 5, 745-752.

Dearden JC. 1985 - Partitioning and lipophilicity in quantitative structure-activity relationships. Environmental Health Perspectives 61, 203-228.

Ertl P, Rohde B, Selzer P. 2000 - Fast calculation of molecular polar surface area as a sum of fragment based contributions and its application to the prediction of drug transport properties. The Journal of Medicinal Chemistry 43, 3714-3717.

Ghannoum MA, Rice LB. 1999 - Antifungal agents mode of action, mechanism of resistance and correlation of these mechanisms with bacterial resistance. Clinical Microbiology Reviews 12, 501-517.

Gleeson MP. 2008 - Generation of a set of simple, interpretable ADMET rules of thumb. The Journal of Medicinal Chemistry 51, 817-834.

Hann MM, Leach AR, Harper G. 2001 - Molecular complexity and its impact on the probability of finding leads for drug discovery. The Journal of Chemical Information and Modeling 41, 856864.

Hann MM, Oprea TI. 2004 - Pursuing the lead likeness concept in pharmaceutical research. Current Opinion in Chemical Biology 8, 225-263.

Hastie T, Tibshirani R, Friedman J. 2009 - Principal components. In: The elements of statistical learning. 2nd ed. Springer, 534-541. 
Hendrickson JB, Huang P, Toczko AG. 1987 - Molecular complexity: a simplified formula adapted to individual atoms. The Journal of Chemical Information and Modeling 27, 63-67.

Jolliffe IT. 2002 - Principal component analysis. 2nd ed. New York: Springer.

Kubinyi H. 1979 - Lipophilicity and drug activity. Progress in Drug Research 23, 97-198.

Kujawski J, Bernard MK, Janusz A, Kuźma W. 2012 - Prediction of log P - ALOGPS Application in Medicinal Chemistry Education. The Journal of Chemical Education 89, 64-67.

Lebart L, Morineau A, Warwick KM. 1984 - Multivariate descriptive statistical analysis: correspondence analysis and related techniques for large matrices. New York: Wiley.

Leeson PD, Davis AM. 2004 - Time-related differences in the physical property profiles of oral drugs. The Journal of Medicinal Chemistry 47, 6338-6348.

Leeson PD. 2016 - Molecular inflation, attrition and the rule of five. Advanced Drug Delivery Reviews 101, 22-33.

Lepesheva GI, Waterman MR. 2004 - CYP51 - the omnipotent P450. Molecular and Cellular Endocrinology 215, 165-170.

Lovering F, Bikker J, Humblet C. 2009 - Escape from flatland: increasing saturation as an approach to improving clinical success. The Journal of Medicinal Chemistry 52, 6752-6756.

Lovering F. 2013 - Escape from Flatland 2: complexity and promiscuity. Medicinal Chemistry Communication 4, 515-519.

Meanwell NA. 2011 - Improving drug candidates by design: a focus on physicochemical properties as a means of improving compound disposition and safety. Chemical Research in Toxicology 24, 1420-1456.

National Center for Biotechnology Information. 2018a - Bitertanol. PubChem Compound Database; CID=91656 https://pubchem.ncbi.nlm.nih.gov/compound/91656 (accessed 18 January 2018).

National Center for Biotechnology Information. 2018b - Cyproconazole. PubChem Compound Database; CID=86132 https://pubchem.ncbi.nlm.nih.gov/compound/86132 (accessed 18 January 2018).

National Center for Biotechnology Information. 2018c - Epoxiconazole. PubChem Compound Database; CID=3317081 https://pubchem.ncbi.nlm.nih.gov/ compound/3317081 (accessed 18 January 2018).

National Center for Biotechnology Information. 2018d - Flusilazole. PubChem Compound Database; CID=73675 https://pubchem.ncbi.nlm.nih.gov/compound/73675 (accessed 18 January 2018).

National Center for Biotechnology Information. 2018e - Hexaconazole. PubChem Compound Database; CID=66461 https://pubchem.ncbi.nlm.nih.gov/compound/66461 (accessed 18 January 2018).

National Center for Biotechnology Information. 2018f - Imazalil. PubChem Compound Database; CID=37175 https://pubchem.ncbi.nlm.nih.gov/compound/37175 (accessed 18 January 2018).

National Center for Biotechnology Information. 2018g - Myclobutanil. PubChem Compound Database; CID=6336 https://pubchem.ncbi.nlm.nih.gov/compound/6336 (accessed 18 January 2018).

National Center for Biotechnology Information. 2018h - Penconazole. PubChem Compound Database; CID=91693 https://pubchem.ncbi.nlm.nih.gov/compound/91693 (accessed 18 January 2018).

National Center for Biotechnology Information. 2018i - Prochloraz. PubChem Compound Database; CID=73665 https://pubchem.ncbi.nlm.nih.gov/compound/73665 (accessed 18 January 2018).

National Center for Biotechnology Information. 2018j - Propiconazole. PubChem Compound Database; CID=43234 https://pubchem.ncbi.nlm.nih.gov/compound/43234 (accessed 18 January 2018).

National Center for Biotechnology Information. 2018k - Tebuconazole. PubChem Compound Database; CID=86102 https://pubchem.ncbi.nlm.nih.gov/compound/86102 (accessed 18 January 2018). 
National Center for Biotechnology Information. 2018l - Triadimefon. PubChem Compound Database; CID=39385 https://pubchem.ncbi.nlm.nih.gov/compound/39385 (accessed 18 January 2018).

National Center for Biotechnology Information. 2018m - Triadimenol. PubChem Compound Database; CID=41368 https://pubchem.ncbi.nlm.nih.gov/compound/41368 (accessed 18 January 2018).

National Center for Biotechnology Information. 2018n - Bifonazole. PubChem Compound Database; CID=2378 https://pubchem.ncbi.nlm.nih.gov/compound/2378 (accessed 18 January 2018).

National Center for Biotechnology Information. 2018o - Clotrimazole. PubChem Compound Database; CID=2812 https://pubchem.ncbi.nlm.nih.gov/compound/2812 (accessed 18 January 2018).

National Center for Biotechnology Information. 2018p - Fluconazole. PubChem Compound Database; CID=3365 https://pubchem.ncbi.nlm.nih.gov/compound/3365 (accessed 18 January 2018).

National Center for Biotechnology Information. 2018q - Itraconazole. PubChem Compound Database; CID=55283 https://pubchem.ncbi.nlm.nih.gov/compound/55283 (accessed 18 January 2018).

National Center for Biotechnology Information. 2018r - Ketoconazole. PubChem Compound Database; CID=456201 https://pubchem.ncbi.nlm.nih.gov/compound/456201 (accessed 18 January 2018).

National Center for Biotechnology Information. 2018s - Miconazole. PubChem Compound Database; CID=4189 https://pubchem.ncbi.nlm.nih.gov/compound/4189 (accessed 18 January 2018).

Pfaller MA, Diekema DJ. 2007 - Epidemiology of invasive candidiasis: a persistent public health problem. Clinical Microbiology Reviews 20, 133-163.

Rekha S, Visyasagar GM. 2013 - Anti-candida activity of medicinal plants. International Journal of Pharmacy and Pharmaceutical Sciences 5, 9-16.

Ritchie TJ, Macdonald SJ. 2009 - The impact of aromatic ring count on compound developabilityare too many aromatic rings a liability in drug design? Drug Discovery Today 14, 1011-1020.

Roy AB, Basak SC, Harris DK, Magnuson VR. 1984 - Neighborhood complexities and symmetry of chemical graphs and their biological activity, in: Avula XJR, Kalman RE, Liapis AI, Rodin EY (Eds.), Mathematical Modelling on Science and Technology, Pergamon Press, New York, $745-750$.

Rozman D, Waterman MR. 1998 - Lanosterol 14alpha-demethylase (CYP51) and spermatogenesis. Drug Metabolism and Disposition 26, 1199-1201.

Sumalapao DEP, Janairo JIB, Gloriani NG. 2018 - Dipole moment, solvation energy, and ovality account for the variations in the biological activity of HIV-1 reverse transcriptase inhibitor fragments. Annual Research \& Review in Biology 22, 1-8.

Trosken ER, Adamska M, Arand M, Zarn JA et al. 2006 - Comparison of lanosterol-14 $\alpha-$ demethylase (CYP51) of human and Candida albicans for inhibition by different antifungal azoles. Toxicology 228, 24-32.

Veber DF, Johnson SR, Cheng HY, Smith BR et al. 2002 - Molecular properties that influence the oral bioavailability of drug candidates. The Journal of Medicinal Chemistry 45, 2615-2623.

Waring MJ. 2010 - Lipophilicity in drugs. Expert Opinion on Drug Discovery 5, 235-248.

Wenlock MC, Austin RP, Barton P, Davis AM, Leeson PD. 2003 - A comparison of physiochemical property profiles of development and marketed oral drugs. The Journal of Medicinal Chemistry 46, $1250-1256$.

Zampieri D, Mamolo MG, Vio L, Banfi E et al. 2007 - Synthesis, antifungal and antimycobacterial activities of new bis-imidazole derivatives, and prediction of their binding to P450(14DM) by molecular docking and MM/PBSA method. Bioorganic \& Medicinal Chemistry 15, 74447458. 
Zonios D, Bennett JE. 2008 - Update on azole antifungals. Seminars in Respiratory and Critical Care Medicine 29, 198-210. 